\title{
Rifapentine Polylactic Acid Sustained-Release Microsphere Complex for Spinal Tuberculosis Therapy: Preparation, in vitro and in vivo Studies
}

\author{
Zhen Wang ${ }^{1,2, *}$ \\ Abulikemu Maimaitiaili ${ }^{2}$ \\ Tengfei Wang ${ }^{2}$ \\ Xinghua Song ${ }^{2,3}, *$ \\ 'Department of Orthopeadics, The \\ Affiliated Linfen Hospital of Shanxi \\ Medical University, Linfen, Shanxi \\ Province, People's Republic of China; \\ ${ }^{2}$ Department of Orthopeadics, The First \\ Affiliated Hospital of Xinjiang Medical \\ University, Urumqi, Xinjiang Province, \\ People's Republic of China; ${ }^{3}$ Department \\ of Orthopeadics, The Affiliated Shunde \\ Hospital of Jinan University, Foshan, \\ Guangdong Province, People's Republic \\ of China \\ *These authors contributed equally to \\ this work
}

Purpose: Spinal tuberculosis has been a common clinical extrapulmonary tuberculosis in recent years. The general anti-tuberculosis drug treatment cycle is long, with unsatisfactory efficacy. This study focused on the preparation and evaluation of rifapentine polylactic acid sustained-release microsphere complex for spinal tuberculosis therapy.

Methods: Rifapentine polylactic acid sustained-release microspheres (RPSMs) were prepared through the double emulsion solvent evaporation method, and RPSMs were combined with hydroxyapatite/ $\beta$-tricalcium phosphate $(\mathrm{HA} / \beta$-TCP) composite material to obtain drugloaded, sustained-release complex. We evaluated the complex for dynamics of drug release and osteogenic ability using in vitro release test, alkaline phosphatase and alizarin red staining, real-time PCR and Western blot. A rabbit model of a spinal tuberculosis defect was established and repaired using HA/ $\beta$-TCP or complex. The ability of anti-tuberculosis and tissue repair effects of the complex were evaluated through in vivo experiments.

Results: The complex constructed of RPSMs and HA/ $\beta$-TCP demonstrated a long drug release time, with no significant inhibition of cell osteogenic differentiation in vitro experiments. Postoperative macroscopic observation, immunohistochemical staining and Nilsson histological scores showed that the complex has good effects on the tissue repair. Moreover, the erythrocyte sedimentation rate (ESR) and C-reactive protein (CRP), important indexes of inflammation, decreased to normal levels in the complex group.

Conclusion: In vitro and in vivo experiments demonstrated that the complex constructed of RPSMs and HA/ $\beta$-TCP effectively treated spinal tuberculosis. Therefore, the complex represents a promising approach for the treatment of spinal tuberculosis.

Keywords: spinal tuberculosis, sustained-release microsphere, antibiotics-loaded bone composite scaffold, rifapentine, tissue engineering

\section{Introduction}

Tuberculosis (TB) is a chronic consuming disease caused by infection with Mycobacterium tuberculosis. The WHO reported that there were 10.0 million TB patients in 2019, of whom 1.4 million died. ${ }^{1}$ Spinal TB is a common extrapulmonary $\mathrm{TB}$, often accompanied by a certain degree of structural damage and deformity, affecting the stability of the spine, and may be accompanied by a series of serious complications such as spinal cord or nerve function damage, ${ }^{2}$ which often require surgical treatment. Long-term medication is needed to prevent recurrence after surgical treatment.

Spinal TB often forms parenchyma and calcified necrosis in the lesions, with a reduced blood supply in the necrotic lesions. It is difficult for traditional anti-TB
Correspondence: Xinghua Song Department of Orthopeadics, The Affiliated Shunde Hospital of Jinan University, Foshan, 528303, People's Email 1787064049@qq.com

Zhen Wang

Department of Orthopeadics, The Affiliated Linfen Hospital of Shanxi Medical University, Linfen, 041000 , People's Republic of China Email 421252955@qq.com 
drug preparations and routes of administration to form effective drug concentrations in necrotic foci, ${ }^{3}$ which cause a series of side effects, ${ }^{4,5}$ leading to decreased patient compliance. To overcome this problem and improve the therapeutic effect of spinal tuberculosis, we developed anti-TB drug sustained-release microspheres, which achieved a longer duration of sustained release and retention.

Polylactic acid is a non-toxic, degradable material with good biocompatibility. It has been approved by the US FDA and the European Food and Drug Administration as a drug delivery carrier. ${ }^{6}$ Rifapentine, one of the rifamycin antibiotics, is a new-generation, long-acting drug that exhibits good antibacterial potency against TB with a minimal inhibitory concentration (MIC) of $0.03-0.06$ $\mu \mathrm{g} / \mathrm{mL}{ }^{7}$ Compared with the classical rifamycin antibiotics, rifapentine exhibits stronger capability to resist hydrolysis, fewer adverse reactions and greater antibacterial effect. ${ }^{8}$ Consequently, we used rifapentine and polylactic acid to prepare the RPSMs.

Spinal TB is characterized by bone defects caused by bone remodeling and an imbalance of bone resorption..$^{9-11}$ Placing the RPSMs on the spinal TB bone defect by surgical treatment would be a feasible method of treatment. We used hydroxyapatite/ $\beta$-tricalcium phosphate (HA/ $\beta$-TCP) composite material which has strong drugloading ability, ${ }^{12}$ good biological activity, is easily degradable, and can promote bone growth, ${ }^{13}$ as carrier-loaded RPSMs, to prepare the complex. These can repair and reconstruct the original bone tissue while treating spinal TB.

In this study, the effects of this complex on stem cell proliferation and osteogenic differentiation were evaluated by in vitro experiments, and the therapeutic and tissuerepairing effects of the complex on a spinal TB defect model were evaluated in vivo.

\section{Materials and Methods}

\section{Animals and Reagents}

Thirty-six mature male New Zealand white rabbits (mean weight: $2.5 \mathrm{~kg}$ ) fed a standard diet were provided by the Experimental Animal Research Center of Xinjiang Uygur Autonomous Region. License number: SCXK (2011-0001). The study was approved by the Animal Ethics Committee of First Affiliated Hospital of Xinjiang Medical University (Approval no. IACUC-20180223-13) and conformed to the "Guide for the Care and Use of Laboratory Animals" published by the Institute of Laboratory Animal Research (Washington, DC, USA). All experiments were performed following relevant named institutional and national guidelines and regulations.

Dulbecco's modified Eagle's medium (Hyclone, Logan, UT, USA), fetal bovine serum (Gibco BRL, Grand Island, NY, USA), osteogenic induction medium (Cyagen Biosciences Co. Ltd., Suzhou, China), Cell Counting Kit-8 (Dojindo, Kumamoto, Japan), Rifapentine (Sichuan Mingxin Pharmaceutical Co. Ltd., Chengdu, China), gelatin (Sigma-Aldrich, St Louis, MO, USA), polylactic acid $(\mathrm{MV}=30,000$, Shandong Medical Devices Co. Ltd., Jinan, China), synthetic hydroxyapatite/ $\beta$-tricalcium phosphate (HA/ $\beta$-TCP) scaffold (composed of $60 \%$ HA and $40 \% \beta$-TCP, Bi-Ostetic, Berkeley, CA, USA), BCIP/NBT chromogenic kit, Alizarin Red dye solution were purchased from Solarbio Co., Ltd (Beijing, China), anti-Collagen I, anti-Osteocalcin and anti-GAPDH antibodies were purchased from Abcam (Cambridge, UK) and Mycobacterium tuberculosis were provided by Xinjiang Academy of Animal Science.

\section{Culture of BMSCs}

BMSCs were isolated from 12-week-old New Zealand white rabbits according to our previous culture method. ${ }^{14}$ BMSCs were cultured in Dulbecco's modified Eagle's medium (DMEM), supplemented with fetal bovine serum $(10 \%, \mathrm{w} / \mathrm{v})$, penicillin $(100 \mathrm{units} / \mathrm{mL})$, and streptomycin $(100 \mu \mathrm{g} / \mathrm{mL})$.

\section{CCK-8 Assay for Cell Survival in vitro}

Three generations of rabbit BMSCs were divided into five groups, which were inoculated into a 96-well plate at a density of $5 \times 10^{3}$ cells $/ \mathrm{mL}$ suspension, three wells per group. The cells were cultured in medium containing different concentrations of rifapentine $(15.0,30.0,45.0$, 60.0 , and $75.0 \mu \mathrm{g} / \mathrm{mL}$ ). The CCK-8 colorimetric method was used to measure cell proliferation every day.

\section{Microsphere Preparation}

Using rifapentine as the raw material, RPSMs were prepared by the double emulsion-solvent evaporation method. First, $0.1 \mathrm{~g}$ of polylactic acid was accurately weighed and dissolved in $5 \mathrm{~mL}$ of dichloromethane. After it was completely dissolved, $0.1 \mathrm{~g}$ of rifapentine was added, cooled to $4^{\circ} \mathrm{C}$, then placed on an ice block and treated with an ultrasonic cell crusher for $1.5 \mathrm{~min}$ to achieve emulsification. After a high-speed stirring at $1500 \mathrm{rpm}$, the emulsion was injected 
into $25 \mathrm{~mL}$ of $0.5 \%$ gelatin aqueous solution through a micro-injection pump, emulsified for $5 \mathrm{~min}$ to form an oil/water $(\mathrm{O} / \mathrm{W})$ emulsion, diluted with $75 \mathrm{~mL}$ of distilled water, and then gently stirred for 5-7 hours to ensure it was evenly mixed. Dichloromethane was volatilized with the microspheres solidified. The microspheres obtained in the suspension were suction-filtered through a water filtration membrane with a pore size of $0.88 \mu \mathrm{m}$, collected, washed with distilled water, centrifuged three times at $800 \times \mathrm{g}$, and then freeze-dried for $24 \mathrm{~h}$. To make blank PLA microspheres for the control group, rifapentine was not added to the dichloromethane, as the only change to the procedure described above.

\section{Physicochemical Properties of RPSMs}

Ultraviolet spectrophotometry was used to measure different concentrations of rifapentine and the standard curve was drawn. Then, $5 \mathrm{mg}$ of RPSMs were weighed, placed in $5 \mathrm{~mL}$ of dichloromethane, sonicated for 20 minutes, centrifuged at $800 \times \mathrm{g}$, and the supernatant was collected. The absorbance was measured and converted into a standard curve equation to obtain the drug concentration. The encapsulation and drug loading efficiency of three batches of prepared microspheres were measured three times and the average encapsulation and drug loading efficiency of the microspheres were calculated. The drug loading efficiency of RPSMs was calculated as the percent ratio of the actual drug content to the total microsphere weight, while the encapsulation efficiency was calculated as the percent ratio of the actual drug loading to the theoretical drug loading. The morphology of the microspheres was observed under a scanning electron microscope (JEOL, Tokyo, Japan). The Ultraviolet-visible (UV-vis) spectra of rifapentine, RPSMs and blank PLA microspheres at a concentration of $1 \mathrm{mg} / \mathrm{mL}$ were measured on a UV-2700 UV-vis spectrophotometer (Shimadzu, Japan).

\section{Preparation of Complex and in vitro Release Test}

First, $3 \mathrm{mg}$ of the RPSMs was uniformly mixed with 100 $\mu \mathrm{L}$ of osteogenic induction medium, and added dropwise to the scaffold (HA/ $\beta$-TCP) to form the complex. The 3rdgeneration stem cells were suspended at a cell density of $3 \times 10^{6}$ cells $/ \mathrm{mL}$; then, $400 \mu \mathrm{L}$ of the stem cell suspension was slowly added dropwise to the complex. The mixture was transferred to a Petri dish after $3 \mathrm{~h}$ in culture, and $5 \mathrm{~mL}$ of osteogenic induction medium was added. After 14 days, the medium was aspirated along the wall of the dish, the cells were washed three times with PBS, fixed with 4\% paraformaldehyde overnight, subjected to stepwise ethanol dehydration, and sprayed with gold. The adhesion and growth of the cells in the scaffold were observed by scanning electron microscopy.

The complex was placed in a Petri dish, with the medium changed and collected every other day. The medium was centrifuged twice at $800 \times \mathrm{g}$, and the supernatant was collected. Then, $2 \mathrm{~mL}$ of solution were taken and the absorbance was measured at a wavelength of $475 \mathrm{~nm}$ by ultraviolet spectrophotometry, and the same concentration of the culture medium was used as a control. Comparing with the standard curve, the concentration of rifapentine was calculated. The experiment was repeated three times. The drug release curve and cumulative percentage of drug release curve were plotted.

\section{Evaluation of the Effects of Drugs on Osteogenic Differentiation of Cells}

The cells were grouped, with $400 \mu \mathrm{L}$ of BMSCs suspension in each group. Groups were as follows: Naive cell group (BMSCs): cells were cultured in normal medium; Induced group (induced BMSCs, IBMSCs): cells were cultured in osteogenic induction medium; RPSMsinduced group (IBMSCs+RPSMs): cells were mixed with $3 \mathrm{mg}$ of RPSMs and cultured in osteogenic induction medium; Complex-induced group (IBMSCs+RPSMs $+\mathrm{HA} / \beta$-TCP): cells were added dropwise to the complex and cultured in osteogenic induction medium. After 21 days of culture, the four groups of cells were trypsinized, counted and plated again, at $1 \times 10^{5} /$ well, and cultured in an incubator for 3 days before being subjected to alkaline phosphatase (ALP) and Alizarin Red staining according to the manufacturer's instructions. The above-mentioned experiments were repeated three times.

\section{Real-Time PCR Analysis of mRNA Expression}

Total RNA was isolated from cells of each group using TRIZOL reagent (Invitrogen, Thermo Fisher Scientific, Waltham, MA, USA) according to the manufacturer's instructions. Total RNA was incubated with DNase to eliminate any residual DNA that might amplify during PCR. The DyNAmo Flash SYBR Green PCR kit (Thermo Fisher Scientific) was used for PCR. The total RNA of each sample was reverse transcribed and amplified using a RevertAid 
First Strand cDNA Synthesis Kit (Thermo Fisher Scientific). A 7500 ABI PRISM Sequence Detector System (Applied Biosystems, Foster City, CA, USA) was used for detection of PCR products. The primer sequences were designed using Primer Premier 5.0 software. All primers were produced by Sangon Biotechnology Ltd (Shanghai, China). The cycling conditions were as follows: $94^{\circ} \mathrm{C}$ for $10 \mathrm{~min} ; 40$ cycles of $94^{\circ}$ $\mathrm{C}$ for $20 \mathrm{~s}, 55^{\circ} \mathrm{C}$ for $20 \mathrm{~s}, 72^{\circ} \mathrm{C}$ for $20 \mathrm{~s}$; a melting step was added to the end of the amplification procedure. There was no nonspecific amplification determined by the melting curve. GAPDH was used as the internal control. The primer sequences were as follows: collagen I (Rabbit): forward primer (5'-CCCAGAAACAGACGACAAACAAC-3'), reverse primer (5'-AACGGCAAAAACAAATCTCCAAA $\left.-3^{\prime}\right)$; osteocalcin (Rabbit): forward primer (5'GCCCTCACTCTTGTCGCCCTGCT-3'), reverse primer (5'-ACCACCTCGCTGCCCTCCCTCTT-3'); GAPDH (Rabbit): forward primer (5'-ACCTGACCTGCCGCC TGGAGAAAGC-3'), reverse primer (5'-GGAGACGACC TGGTCCTCGGTGTAG-3').

\section{Protein Extraction and Western Blot}

The cells of each group were extracted and lysed with RIPA buffer (Sigma-Aldrich) on ice. The supernatant was collected after centrifugation at $12,000 \times \mathrm{g}, 4^{\circ} \mathrm{C}$ for $30 \mathrm{~min}$. The protein concentration was determined using the BCA (bicinchoninic acid) protein assay kit according to the manufacturer's protocol (biosharp, Hefei, China). Protein samples $(20 \mu \mathrm{g})$ were loaded and electrophoresed on SDS-polyacrylamide gels, transferred to a polyvinylidene difluoride (PVDF) membrane (Roche, Mannheim, Germany), blocked with 5\% skim milk, and incubated overnight at $4^{\circ} \mathrm{C}$ with primary antibodies against collagen I, osteocalcin and GAPDH (dilution, 1:1000, Abcam). Then, the membranes were incubated with goat horseradish peroxidase-conjugated secondary antibody (dilution, 1:2000, Abcam) for 1 hour at room temperature. Protein band signals were developed using an enhanced chemiluminescence (ECL) reagent (Thermo Fisher Scientific) in a gel imaging system (Image Quant 300/RT ECL; GE Healthcare, Chicago, IL, USA). The resulting bands were quantified using ImageJ software, and band intensity was normalized to that of GAPDH.

\section{Establishment and Grouping of Animal Model}

Animal model with spinal TB were established and treated. All the surgical procedures were approved and performed by the Animal Care and Experiment Committee of Xinjiang Medical University. Thirty-six male rabbits were anesthetized with $2 \%$ pentobarbital sodium $(30 \mathrm{mg} / \mathrm{kg})$ via the ear vein, and vertebral bodies 4 and 5 were exposed at the waist. A stainless-steel drill bit was used to drill a bone defect $(3 \mathrm{~mm}$ in diameter and depth) at the proximal transverse process root of the fifth lumbar vertebral body, and $0.1 \mathrm{~mL}$ of $1 \times 10^{6} \mathrm{cfu} / \mathrm{mL}$ TB suspension (H37Rv ATCC 27294) was slowly infused in. One month later, the diseased vertebral body was exposed layer by layer, with the extent of abscess and bone destruction observed, to determine whether the spinal TB model was successfully constructed. Then, the dead bone and paravertebral abscess were scraped off. The rabbits were divided into three groups: Control (no scaffold material) group, HA $/ \beta$-TCP group, and Complex (RPSMs $+\mathrm{HA} / \beta$ TCP) group, with twelve rabbits in each group. Finally, the defect site was sealed with bone wax and the wound was sutured with layered suture.

\section{Biosafety Assessment of the Complex}

The complex was placed in the vertebral bone defect of six male rabbits; the method was same as above. Blood samples were taken before operation (Preoperative) and 1,2,3,4 weeks after operation (Week 1, 2, 3, 4). The general toxicities of the complex to the liver and kidney were assessed by analyzing several key serum parameters at each time point. These assays included aspartate transaminase (AST), alanine transaminase (ALT), blood urea nitrogen $(\mathrm{BUN})$ and creatinine $(\mathrm{Cr})$, which were determined by using the commonly used kits in clinical diagnostic laboratory (Sichuan Maccura Biotechnology, Chengdu, China).

\section{Therapeutic Assessment of the Complex}

Blood was taken before the tuberculosis infection (pre-TB infection), 4 weeks after tuberculosis infection ( $4 \mathrm{w}$ post-TB infection), 6 and 12 weeks after operation ( $6 \mathrm{w}$ postoperation, $12 \mathrm{w}$ post-operation) for determination of erythrocyte sedimentation rate (ESR) by an automatic ESR analyzer (Electa-lab, Forli, Italy). Blood was collected via the ear vein. After the blood was centrifuged, the level of serum C-reactive protein (CRP) was analyzed using a rabbit C Reactive Protein enzyme-linked immunosorbent assay (ELISA) Kit (Abcam) according to the manufacturer's instructions. Absorbance was measured at $450 \mathrm{~nm}$ with pathlength and wavelength correction using Multiskan spectrum (Thermo Fisher Scientific). 


\section{Macroscopic Observations and Immunohistochemical Analyses}

Rabbits were sacrificed by intravenous injection of sodium pentobarbital at 6 and 12 weeks after surgical treatment. Then, the lesion segment and the upper and lower normal segments were removed. After gross examination, the samples were fixed in formalin, decalcified and then embedded in paraffin and cut into $5 \mathrm{~mm}$ sections. The expression of osteocalcin in the regenerated tissue was analyzed by immunohistochemical staining and the positive area is bone tissue. Sections prepared as described above, were dewaxed into xylene and hydrated through graded alcohol. Endogenous peroxidase activity was blocked with $0.3 \%$ hydrogen peroxide in PBS. After blocking with goat serum (dilution, 1:100), sections were incubated with primary antibodies against osteocalcin (dilution, 1:200, Abcam) overnight at $4^{\circ} \mathrm{C}$. After washing by PBS 3 times, secondary goat anti-mouse IgG (dilution, 1:500, Abcam) was added, followed by incubation for 1 $\mathrm{h}$ at $37^{\circ} \mathrm{C}$. Staining was developed in DAB solution, with counterstaining by hematoxylin. The regenerated tissue in the defects was evaluated independently by three observers using the Nilsson histological scores (NHS) ${ }^{15}$

\section{Statistical Analysis}

All data were analyzed using the SPSS 22.0 software (IBM Corporation, Armonk, NY, USA). A one-way ANOVA was followed by a post-hoc Tukey's test to determine the significant differences between the quantitative variables. Differences were considered statistically significant at $\mathrm{p}<0.05$.

\section{Results}

\section{Effect of Rifapentine on Cell Proliferation}

CCK-8 assays were applied to investigate the effect of rifapentine on the viability of BMSCs. As shown in the figure, the results indicated that administration of $\leq 45.0$ $\mu \mathrm{g} / \mathrm{mL}$ rifapentine had no significant inhibitory effect on the proliferation of BMSCs (Figure 1). Therefore, $45.0 \mu \mathrm{g} /$ $\mathrm{mL}$ rifapentine was selected for further experiments.

\section{Preparation and Physicochemical Properties of Microspheres}

The microspheres were red in color, powdery, uniform and non-adhesive. Under an electron microscope, they appeared spherical in shape with a smooth surface and small pores on the surface of individual microspheres (Figure 2A). Using the standard curve equation, the encapsulation and drug loading efficiency of the microspheres were calculated from three batches of prepared microspheres, and the average encapsulation and drug loading efficiency of the microspheres were $80.23 \pm 0.69 \%$ and $35.71 \pm 2.07 \%$, respectively. As shown in Figure 2B, both RPSMs and rifapentine exhibited characteristic absorption at $475 \mathrm{~nm}$, confirming the successful loading of rifapentine.

\section{Preparation and Drug Release Curve of the Complex}

The structure of the complex was observed under an electron microscope. Cell growth on the scaffold material surface was good, with cells having a spindle-shaped morphology, and cellular pseudopods extending around the microspheres (Figure 2C).

The absorbance was used to calculate the drug concentration. The drug release curve and cumulative percentage of drug release curve were plotted (Figure 3). The amount of drug released by the complex on the sixth day reached the highest at $0.0311 \pm 0.0003 \mathrm{mg} / \mathrm{mL}$ before a smooth decrease. On the fifty-eighth day, the amount of drug released was $0.0013 \pm 0.0003 \mathrm{mg} / \mathrm{mL}$, which was significantly higher than the MIC. And the cumulative release amount reached $81.92 \%$. The concentration could not be measured after fifty-eight days.

\section{Evaluation of the Effects of Drugs on Osteogenic Differentiation of Cells}

Under a light microscope (BX53, Olympus, Tokyo, Japan), the ALP staining of four groups showed that the naive cell group was negative, but positive in the other three groups, with no significant difference among the groups, indicating that the BMSCs had differentiated into osteoblasts (Figure 4). For the Alizarin Red staining, the observed results were consistent with the ALP staining.

The mRNAs of collagen I and osteocalcin were analyzed in each group of cells by Real-time PCR. The results indicated that, compared with the RPSMs-induced group, the complex significantly increased the mRNA expression levels of both collagen I and osteocalcin. Notably, the results showed that the expression of both collagen I and osteocalcin mRNA decreased in BMSCs in the presence of RPSMs compared to the induced group (Figure 5A). To further analyze the effects of RPSMs on osteogenic differentiation of cells, Western blot was employed to detect both collagen I and osteocalcin proteins in BMSCs. The complex enhanced 


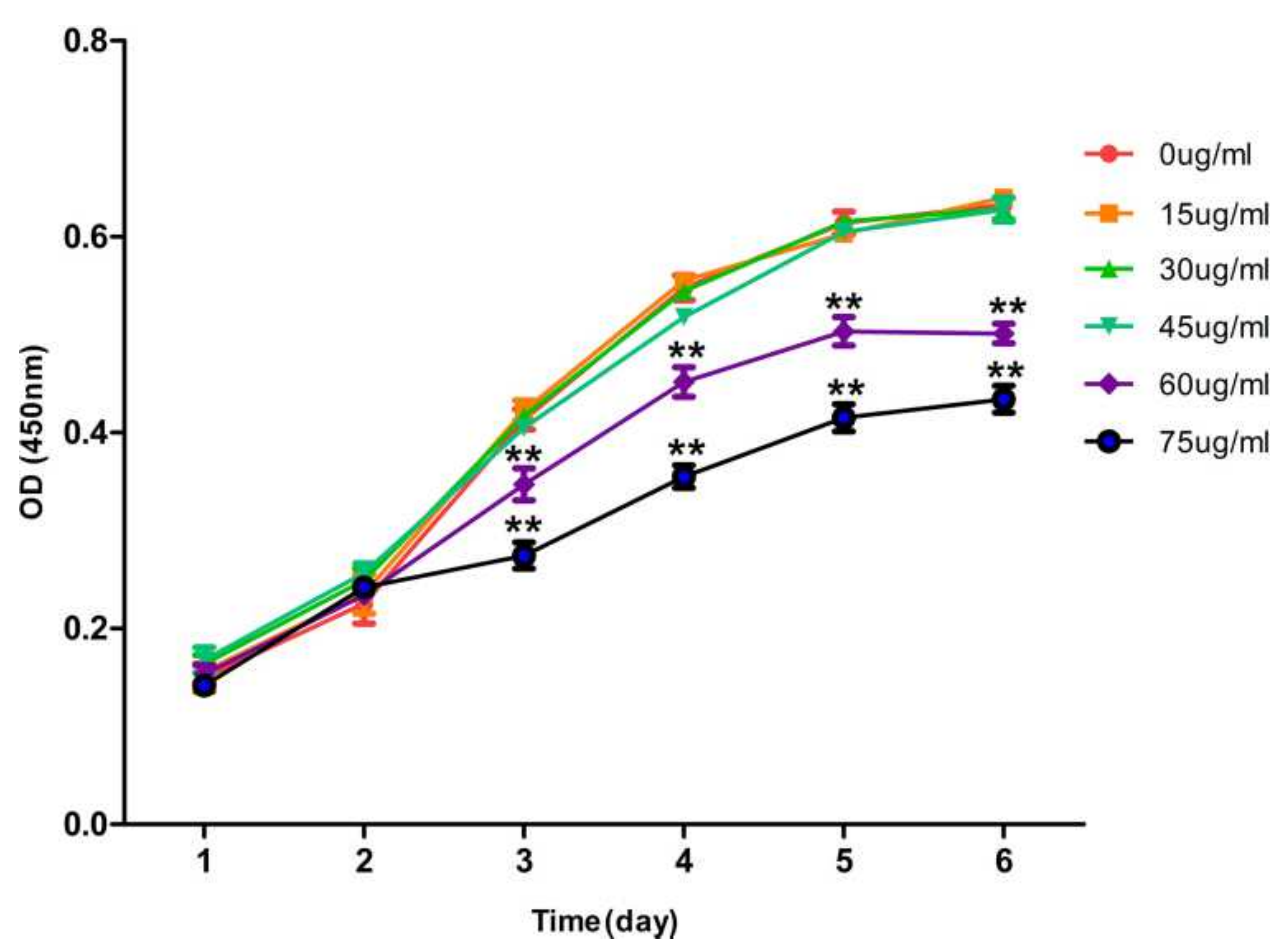

Figure I Effects of different concentrations of rifapentine on the proliferation curve of BMSCs.

Notes: The values presented were the mean \pm SD of three independent experiments. ${ }^{*} * \mathrm{vs} 0 \mu \mathrm{g} / \mathrm{mL},{ }^{*} * \mathrm{P}<0.0 \mathrm{l}$.

Abbreviation: BMSCs, bone marrow mesenchymal stem cells.

the levels of collagen I and osteocalcin proteins when compared with those in the other three groups, whereas RPSMs decreased the levels of collagen I and osteocalcin proteins compared to the induced group (Figure 5B and C).

\section{Biosafety Assessment of the Complex}

The serum biomarkers (AST, ALT, BUN, Cr) of liver and kidney were detected, and there were no significant differences in these biomarkers when compared with that of the pre-operative's $(p>0.05)$. Thus, these toxicological results demonstrate an acceptable biosafety profile of the complex for in vivo use (Table 1).

\section{Evaluation of the Therapeutic Effect of the Complex}

The ESR of the three groups was significantly accelerated at 4 weeks post-TB infection due to the presence of tuberculosis. The ESR gradually decreased in the complex group, and 12 weeks after operation, it was close to preTB infection levels. In contrast, the ESR decreased only
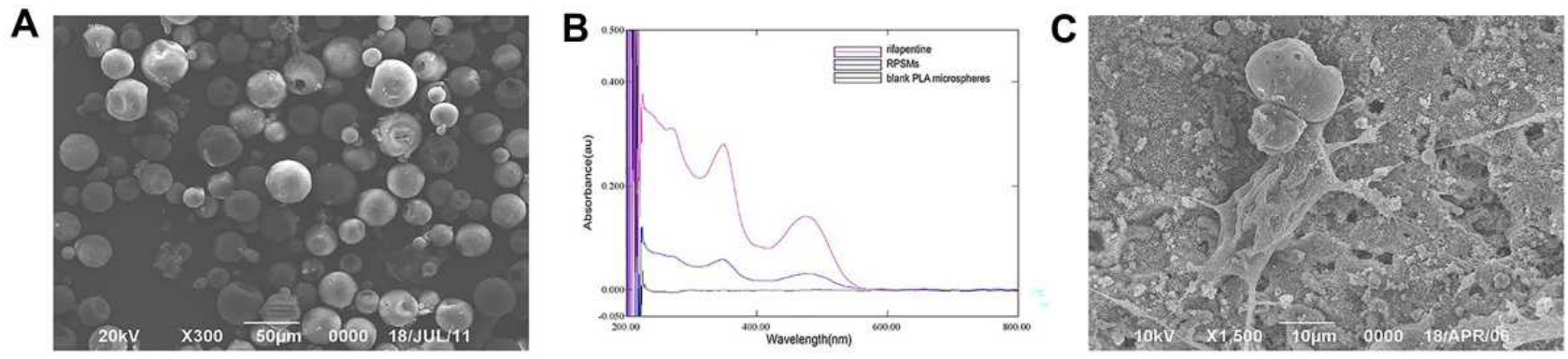

Figure 2 The properties of RPSMs in vitro and the growth of the BMSCs in the RPSMs complex.

Notes: (A) Representative scanning electron micrograph of RPSMs (300x magnification, scale bar $=50 \mu \mathrm{m}$ ); (B) UV-vis spectra of rifapentine, RPSMs and blank PLA microspheres. (C) Representative scanning electron micrograph of BMSCs adhesive growth in the complex $(1500 \times$ magnification, scale bar $=10 \mu \mathrm{m})$

Abbreviations: BMSCs, bone marrow mesenchymal stem cells; RPSMs, rifapentine polylactic acid sustained-release microspheres. 

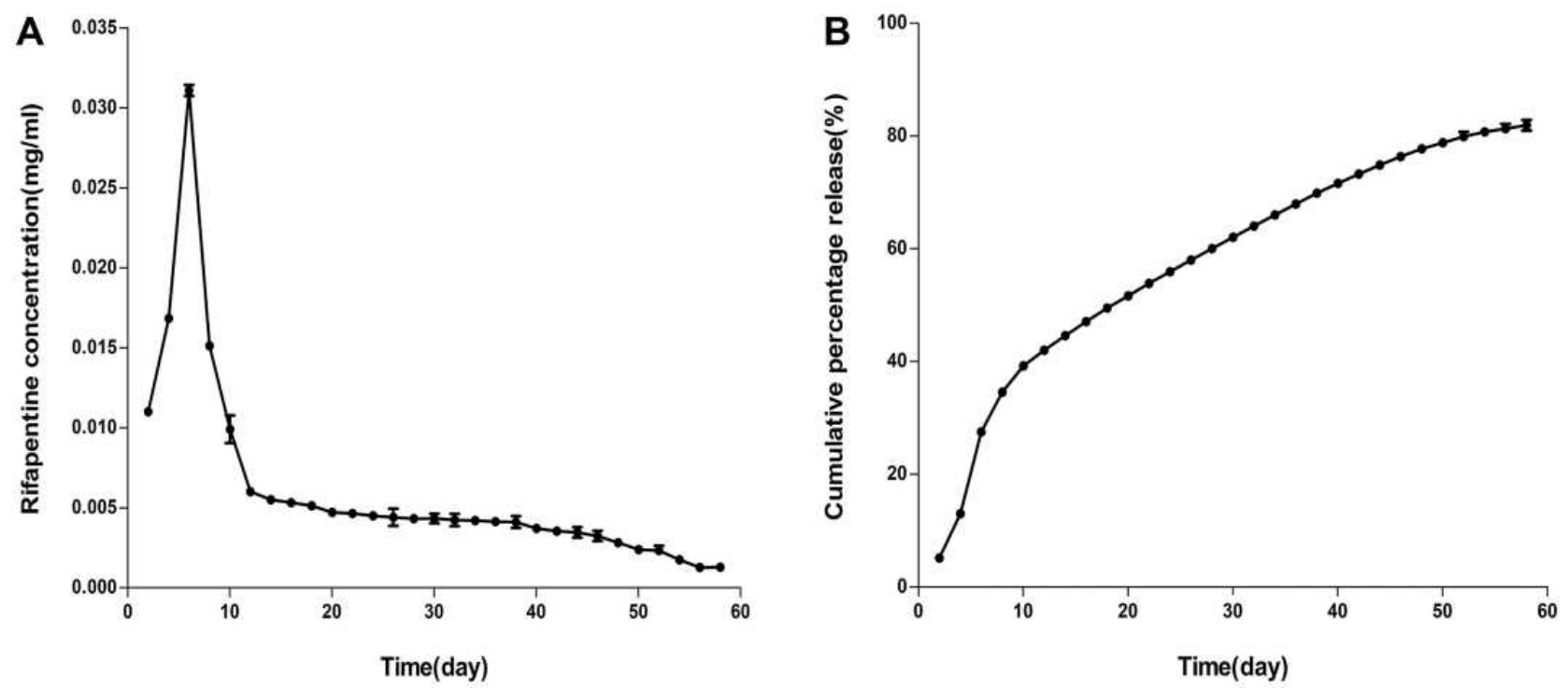

Figure 3 (A) In vitro rifapentine release curve. (B) In vitro cumulative percentage release curve of rifapentine. Note: The values presented were the mean \pm SD of three independent experiments.

slowly in the HA/ $\beta$-TCP group and Control group, but it was still higher than pre-TB infection levels at postoperative week 12 (Figure 6A).

At 4 weeks post-TB infection, the level of CRP was clearly high in the rabbit venous blood test because of the existence of tuberculosis. At 6 weeks after operation, the amount of CRP in the three groups was still significantly different compared with the pre-TB infection levels. The CRP gradually decreased in the complex group, and 12 weeks after operation, it was close to pre-TB infection levels. In the other two groups, it slowly decreased and was still higher than pre-TB infection levels (Figure 6B).

\section{Macroscopic Observations and Immunohistochemical Findings}

The repair of vertebral body defects was observed at 6 and 12 weeks after operation. As shown in Figure 7, after 6 weeks, the spine defect area was extensive with severe surrounding bone destruction and a sinus in the Control group. In the HA/ $\beta$-TCP group, there was no obvious bone defects or sinus. Compared with Control and HA/ $\beta$-TCP groups, the defect area was filled with well-integrated osteoid tissue and bone tissue in the complex group. When it came to 12 weeks, the previous defect was filled with considerable white soft fibrous-like tissue in the Control and HA/ $\beta$-TCP groups. However, the defect area in the complex group was completely replaced by regenerated bone tissue (Figure 7).
At 6 and 12 weeks after operation, specimens were collected from the bone tissue lesion and stained using immunohistochemical techniques of osteocalcin. As shown in Figure 8, at 6 weeks, the bone defect area was filled with fibrous tissue in the Control and HA/ $\beta$-TCP groups. However, the bone defect area was filled with more bone tissue in the complex group. After 12 weeks, the defects of Control and HA/ $\beta$-TCP groups were covered by considerable fibrous tissue. Compared with Control and HA/ $\beta$-TCP groups, it exhibited that more bone tissue and osteoid formation in the complex group (Figure 8).

\section{Histological Scores}

The difference in scores between the complex group and the other two groups was significant at 6 and 12 weeks after operation (Figure 9). The complex group demonstrated the highest score and the control group had the lowest score. The scores of each group increased with time.

\section{Discussion}

Spinal TB is a common extrapulmonary form of $\mathrm{TB}$, which often causes bone destruction and nerve function damage. Surgery is the main mode of treatment for spinal TB. Surgical treatment can remove the lesion tissue, relieve spinal cord or nerve root compression, and reshape the bone for stability. ${ }^{16}$ However, the location of spinal TB is problematic, as drugs cannot directly reach the lesion, often leading to TB recurrence and multidrug-resistant 


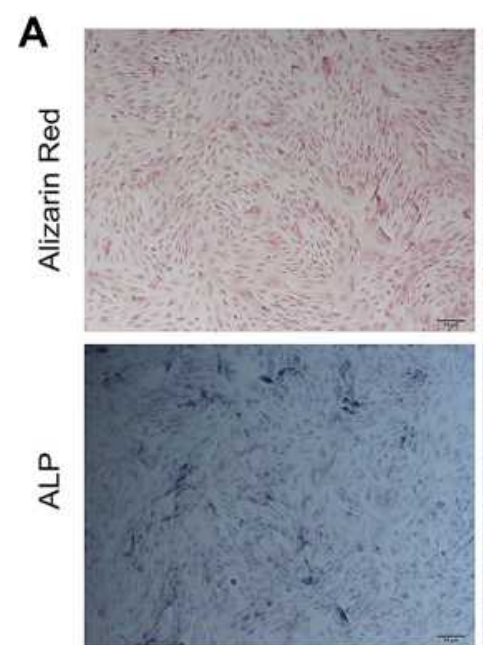

BMSCs
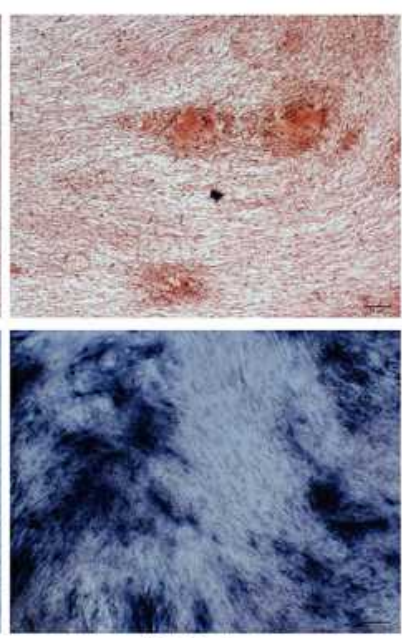

IBMSCs
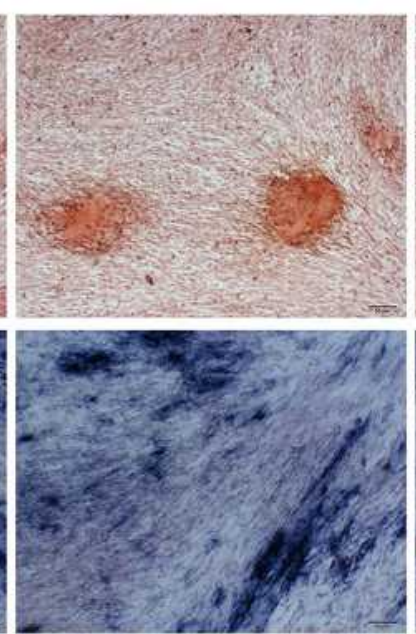

IBMSCs+RPSMs

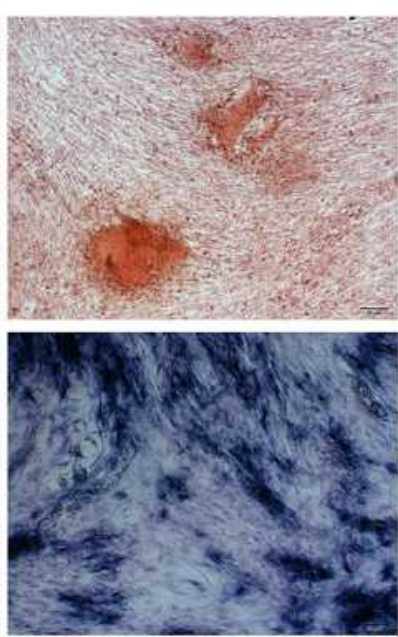

IBMSCs+RPSMs+ HA/ $\beta-T C P$

\section{B}
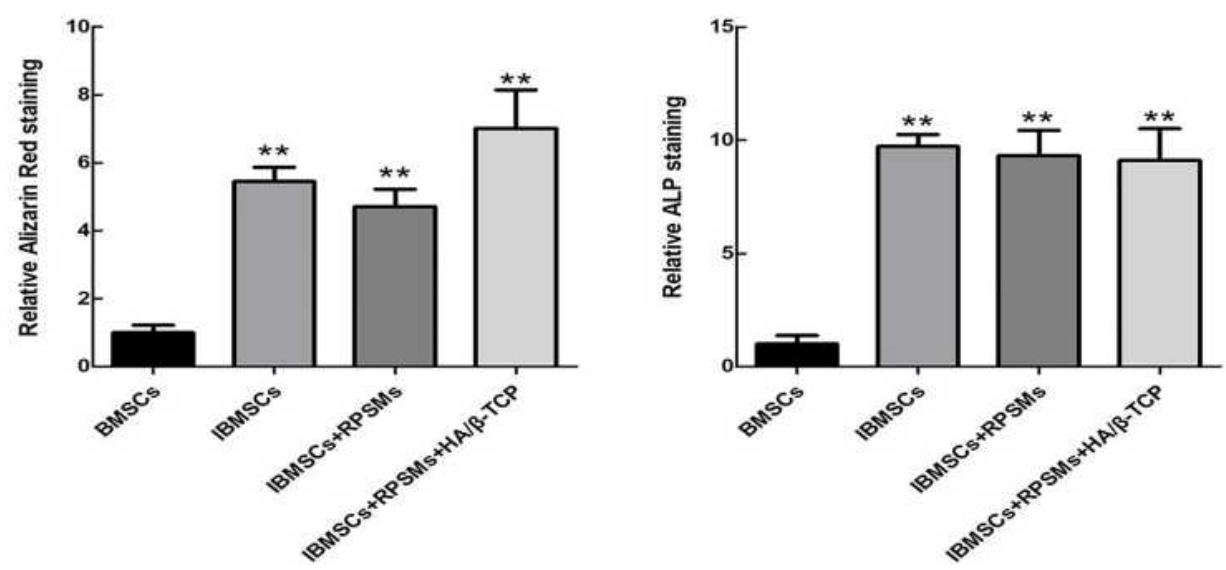

Figure 4 The effects of drugs on osteogenic differentiation of BMSCs were conducted by the Alizarin Red and ALP staining after 21 days of culture.

Notes: (A) Representative Alizarin Red and ALP staining images of the cells ( $100 \times$ magnification, scale bar $=50 \mu \mathrm{m})$. (B) Quantification of stained area of cells in each group. The stained area in cells was quantified using ImageJ software. Above results were obtained from three independent experiments. All data were presented as the mean \pm SD. **vs the BMSCs group, **P<0.01.

Abbreviations: BMSCs, bone marrow mesenchymal stem cells; IBMSCs, induced bone marrow mesenchymal stem cells; RPSMs, rifapentine polylactic acid sustained-release microspheres; HA/ $\beta$-TCP, hydroxyapatite/ $\beta$ - tricalcium phosphate; ALP, alkaline phosphatase.

$\mathrm{TB},{ }^{17}$ and anti-TB drugs should be used. Our novel complex is prepared from RPSMs and HA/ $\beta$-TCP, and placed directly into the lesion site, which not only fills the bone defect of the lesion but also increases the local drug concentration and exerts a long-term stable anti-TB effect, reducing adverse reactions caused by systemic medication.

In recent years, drug delivery systems, such as liposomes and microspheres, have attracted much attention. Since the membrane of liposomes is soft and easily ruptured, the sustained release of liposomes is not satisfactory, ${ }^{18}$ and microspheres were selected as drug delivery system. The microspheres prepared in this study have a higher drug loading efficiency, with encapsulation efficiency fulfilling the requirements of the pharmacopoeia, which proves the reliability of the microsphere preparation process. We cultured the complex together with stem cells in osteogenic induction medium. The drug release peak was found to be $31.6 \mu \mathrm{g} / \mathrm{mL}$ by drug release curve, which was then gradually stabilized, consistent with the general law of drug release. ${ }^{19,20}$ The effects of different concentrations of rifapentine on cell proliferation were investigated by $\mathrm{CCK}-8$ assay at the early stage, and it was found that drug concentrations up to $45.0 \mu \mathrm{g} / \mathrm{mL}$ had no effect on cell proliferation. This indicates that the microspheres have little effect on cell proliferation during drug release. Moreover, the previous study on drug release characteristics in vivo showed that rifapentine maintained above the MIC for 60 days in the 
A

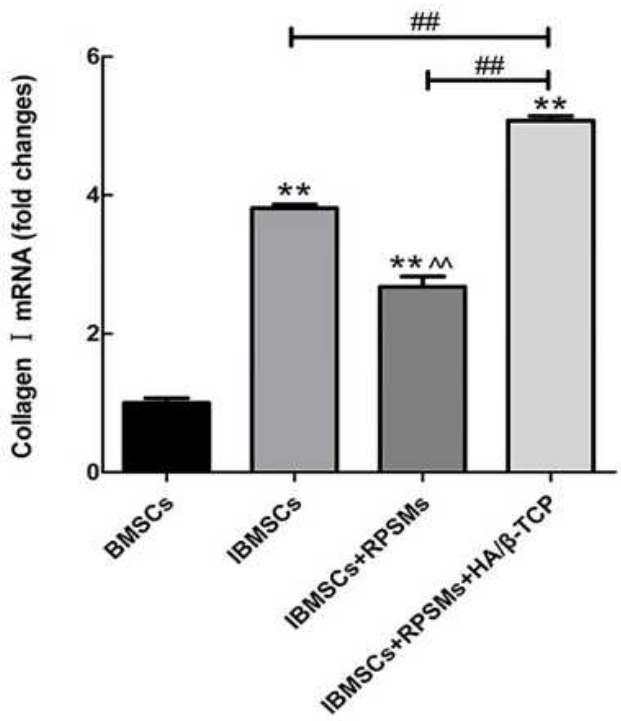

B Collagen
GAPDH

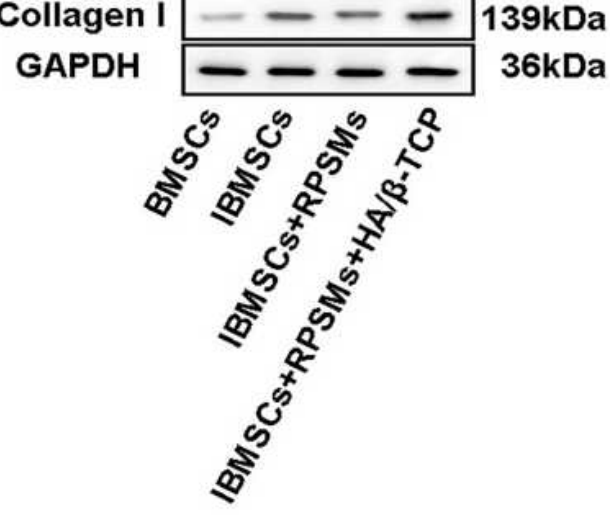

C

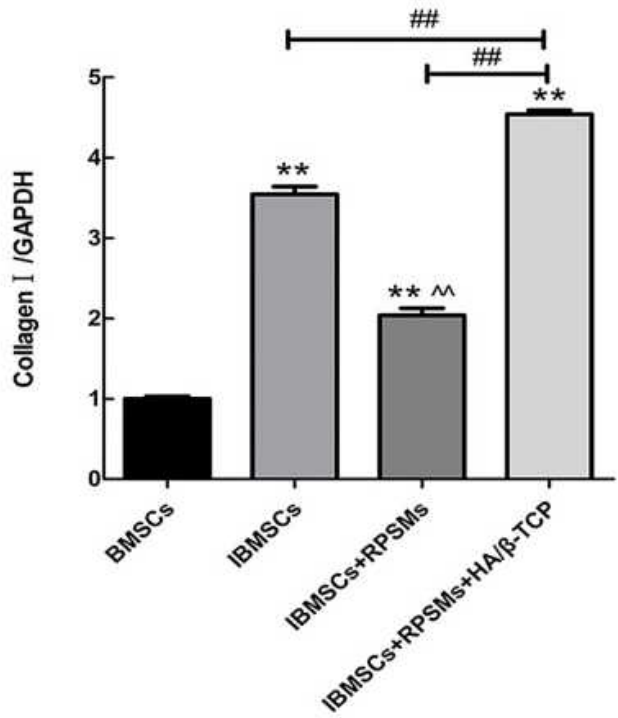

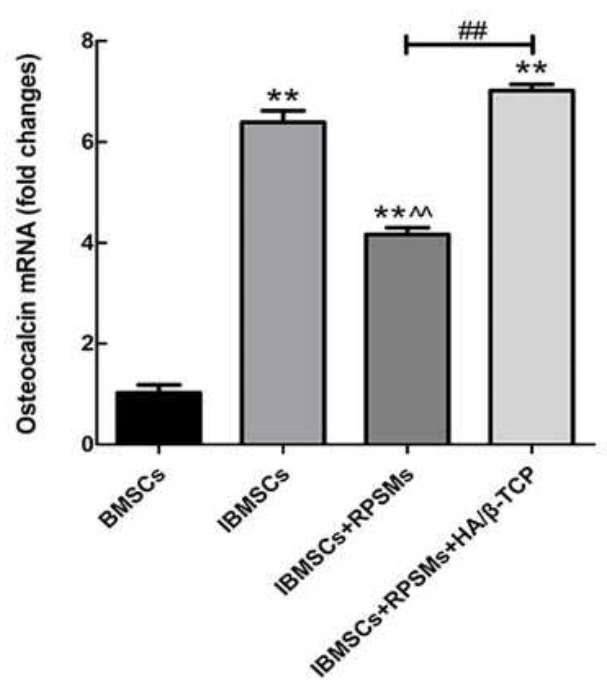
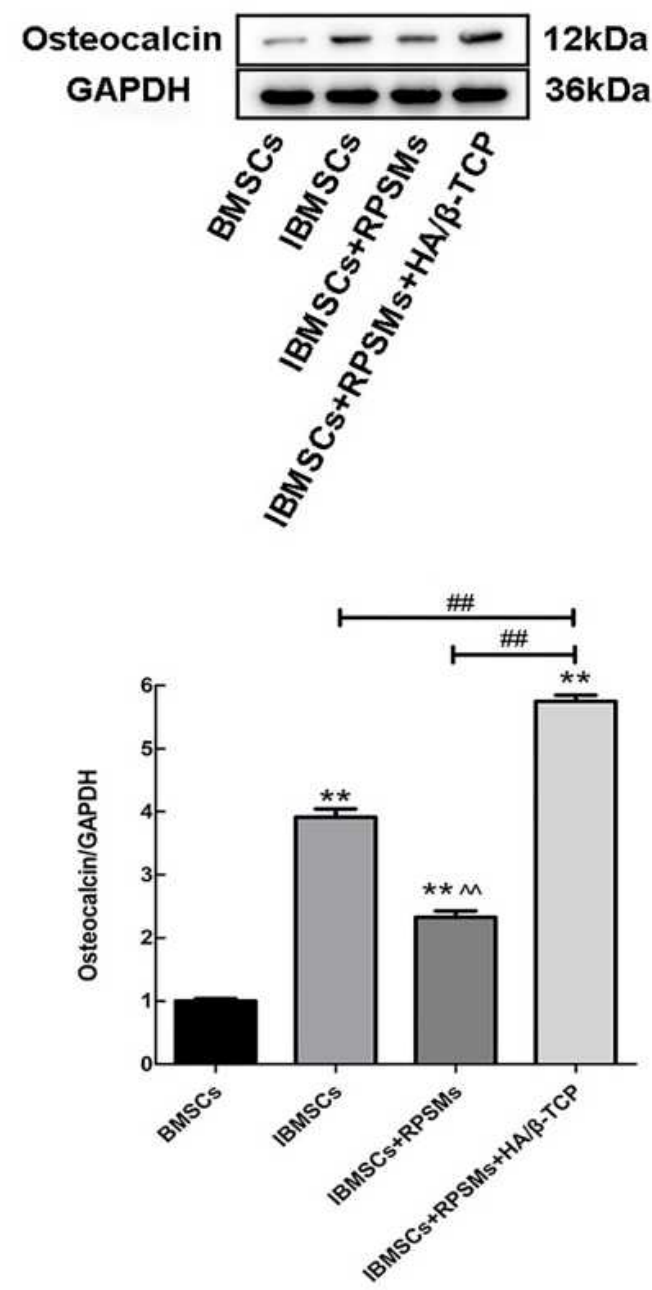

Figure 5 Effects of complex on osteogenic marker collagen I and osteocalcin of four groups of cells were analyzed by Real-time PCR and Western blot in vitro. Notes: (A) The expressions of osteogenic marker genes collagen I and osteocalcin were conducted by Real-time PCR. (B) The protein expressions of collagen I and osteocalcin in BMSCs were detected by Western blot. (C) The relative intensity of each band was quantitated using ImageJ software. GAPDH was employed as a loading control. Above results were obtained from three independent experiments. The data were presented as mean \pm SD. $* * v s$ the BMSCs group, $* * P<0.0 \mathrm{I} ;{ }^{\wedge}$ vs the IBMSCs group, ${ }^{\wedge} \mathrm{P}<0.01 ;$

Abbreviations: BMSCs, bone marrow mesenchymal stem cells; IBMSCs, induced bone marrow mesenchymal stem cells; RPSMs, rifapentine polylactic acid sustained-release microspheres; HA/ $\beta$-TCP, hydroxyapatite/ $\beta$ - tricalcium phosphate. 
Table I Serological Biochemical Markers in the Complex Group at Different Time Points (Mean \pm SD, $n=6$ )

\begin{tabular}{|l|c|c|c|c|c|}
\hline Biomarkers & Preoperative & Week I & Week 2 & Week 3 & Week4 \\
\hline AST (U/L) & $26.00 \pm 4.71$ & $25.50 \pm 5.46$ & $27.1 I \pm 6.54$ & $23.98 \pm 5.33$ & $25.05 \pm 5.38$ \\
ALT (U/L) & $46.62 \pm 13.64$ & $46.93 \pm 11.61$ & $51.33 \pm 12.80$ & $47.58 \pm 10.86$ & $43.15 \pm 16.08$ \\
BUN (mmol/L) & $5.63 \pm 3.34$ & $6.31 \pm 3.17$ & $6.00 \pm 2.88$ & $5.82 \pm 2.20$ & $5.74 \pm 2.31$ \\
Cr $(\mu \mathrm{mol} / \mathrm{L})$ & $63.17 \pm 16.54$ & $57.67 \pm 20.36$ & $61.50 \pm 20.59$ & $64.83 \pm 18.81$ & $56.67 \pm 15.12$ \\
\hline
\end{tabular}

Abbreviations: AST, aspartate aminotransferase; ALT, alanine transaminase; BUN, blood urea nitrogen; $\mathrm{Cr}$, creatinine.

local vertebrae tissue, ${ }^{21}$ which was consistent with the results of in vitro release test. The above results suggested that RPSMs can be stably released in vitro and in vivo.

In the present study, qualitative study (ALP, Alizarin Red staining) and quantitative study (real-time PCR, Western blot) were used to investigate the effects of RPSMs and complex on the osteogenic differentiation of stem cells. ALP and Alizarin Red staining results suggested that RPSMs and complex had limited effects on osteogenic differentiation of stem cells. However, realtime PCR and Western blot were used to analyze the expression levels of both collagen I and osteocalcin, and the results showed that the RPSMs affected the osteogenic differentiation of BMSCs by down-regulating the expression of mRNA and proteins. Rifampicin and rifapentine have the same antibacterial spectrum and they are all rifamycin antibiotics. A number of studies have shown that the osteogenic differentiation of stem cells was inhibited to varying degrees with the increase in rifampicin concentration. $^{22-24}$ The present study indicated that rifapentine was related to the inhibition of osteogenic differentiation of BMSCs.

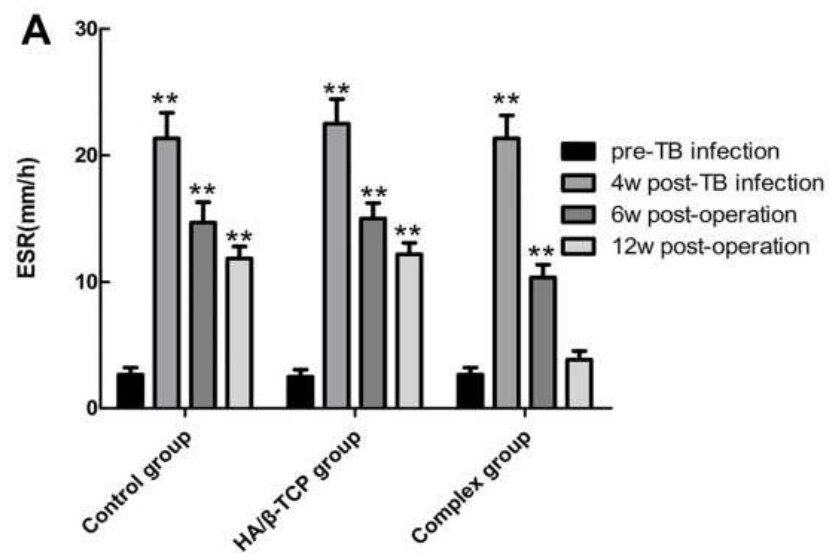

HA $/ \beta$-TCP is a commonly used bone graft material in clinic with good biocompatibility and bone conduction properties, ${ }^{25}$ and previous studies have found that HA/ $\beta$ TCP scaffolds can promote osteogenic differentiation of BMSCs. ${ }^{26-28}$ This study found that the expression of both collagen I and osteocalcin increased in the complexinduced group, indicating that the complex, prepared from RPSMs and HA/ $\beta$-TCP composite material, had promotion effects on osteogenic differentiation of BMSCs. The above results showed that HA/ $\beta$-TCP composite material played an important role in promoting the osteogenic differentiation of BMSCs.

Rabbit spinal TB models are the best model to evaluate drug efficacy for bone TB. ${ }^{29,30}$ We established a rabbit spinal TB model for further study of the complex. A clinically widely used synthetic bone graft material (HA/ $\beta-\mathrm{TCP})$ was used as the experimental control. Macroscopic observation, histological examination and Nilsson histological scores revealed that the repair effect of the complex group was significantly higher than that of the other two groups. These results suggested that treatment with complex promoted defect repair in spinal

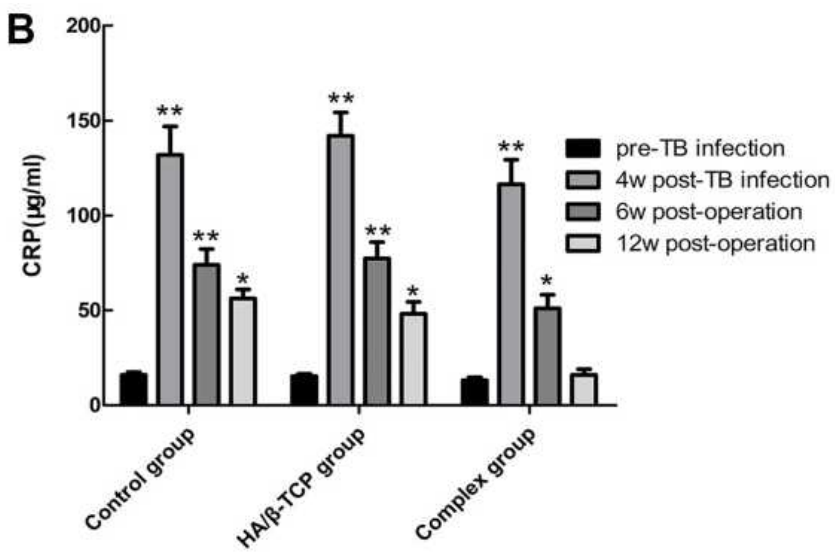

Figure 6 The changes of serum ESR $(\mathbf{A})$ and CRP $(\mathbf{B})$ in each group at different time were observed.

Notes: The results are the mean \pm SD of an experiment performed in triplicate $(n=6)$. ${ }^{*}, * * v s$ pre-TB infection, $* P<0.05, * * P<0.01$

Abbreviations: ESR, the erythrocyte sedimentation rate; CRP, C-reactive protein; HA/ $\beta$-TCP, hydroxyapatite/ $\beta$ - tricalcium phosphate. 


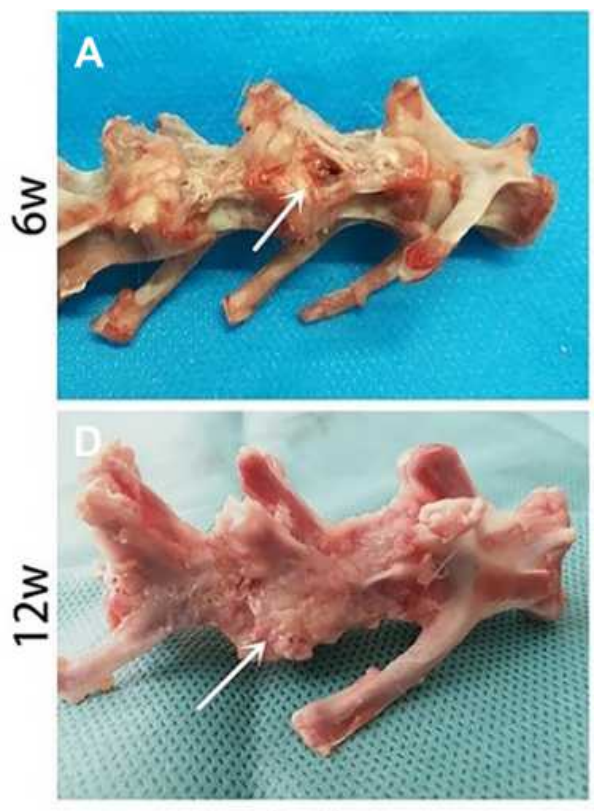

Control group
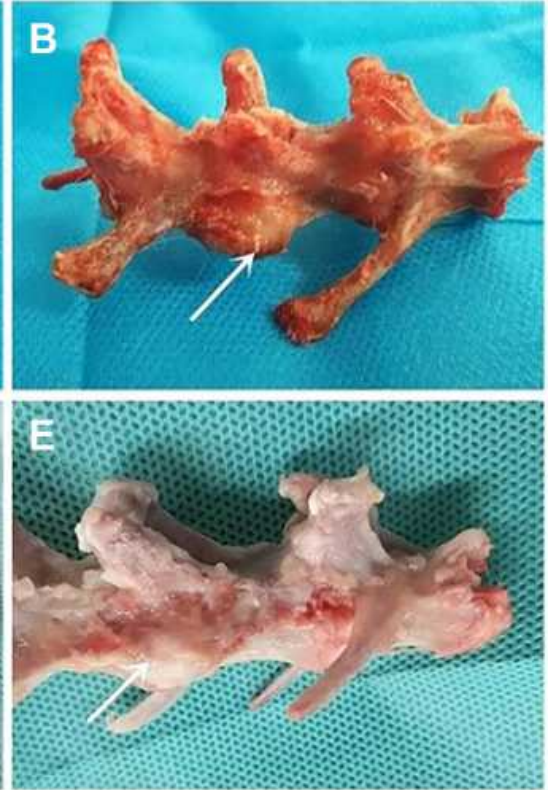

HA/ $\beta$-TCP group

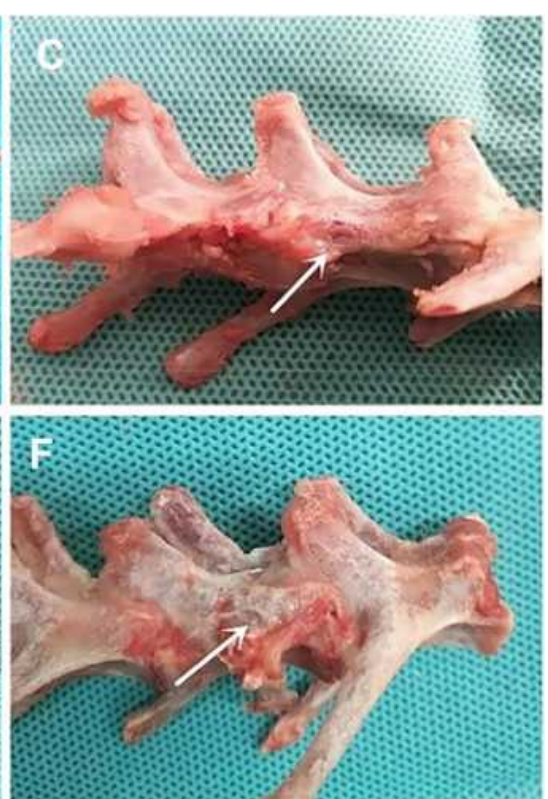

Complex group

Figure 7 Macroscopic observations of the vertebral body defect healing in the three groups at 6 weeks (A-C) and I2 weeks (D-F) after surgery. The white arrow of picture is pointing to the spine defect area.

Abbreviation: HA/ $\beta-\mathrm{TCP}$, hydroxyapatite/ $\beta$ - tricalcium phosphate.
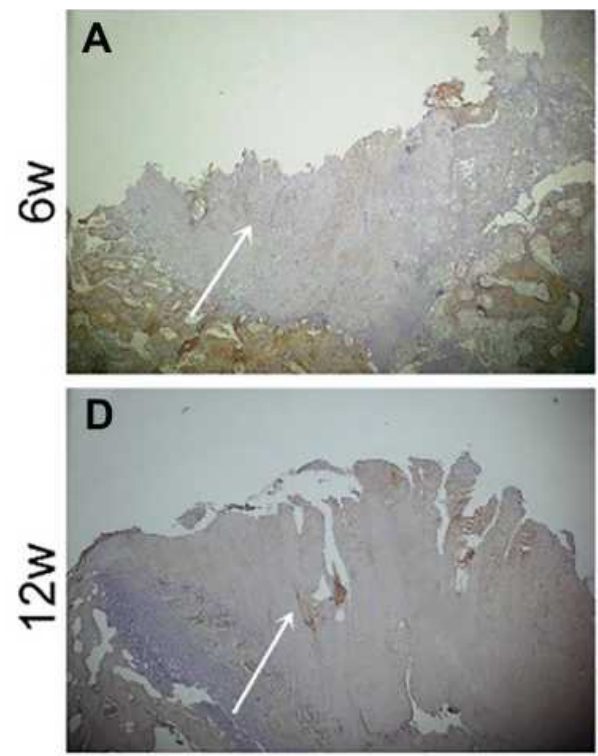

Control group
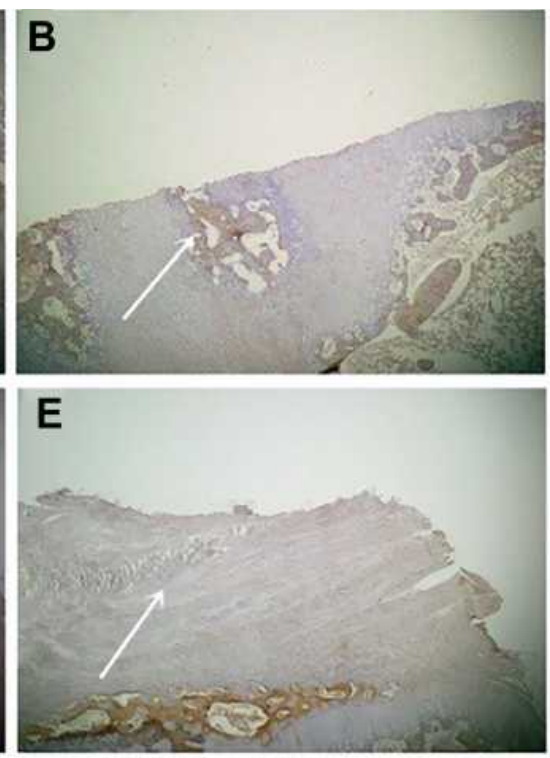

HA/ $\beta-T C P$ group
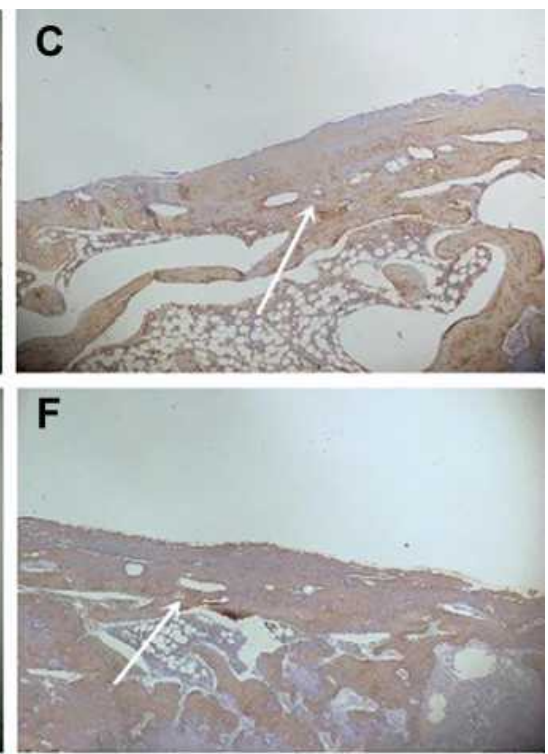

Complex group

Figure 8 The images of osteocalcin immunohistochemical staining from three groups at 6 weeks (A-C) and 12 weeks (D-F) after surgery (40X magnification, scale bar $=200 \mu \mathrm{m})$. The white arrow of picture is pointing to the surface of the spine defect area.

Abbreviation: $\mathrm{HA} / \beta-\mathrm{TCP}$, hydroxyapatite/ $\beta$ - tricalcium phosphate.

tuberculosis model. Furthermore, the changes in the levels of ESR and CRP were measured. ESR and CRP are important indexes of inflammation and stress. If ESR and CRP fall quickly, it indicates that inflammation is improving; however, if they fall slowly or are continuously high, it indicates that inflammation is persisting. ${ }^{31}$ When the ESR and CRP were analyzed in each group, the results showed that both the ESR and CRP in the complex group 


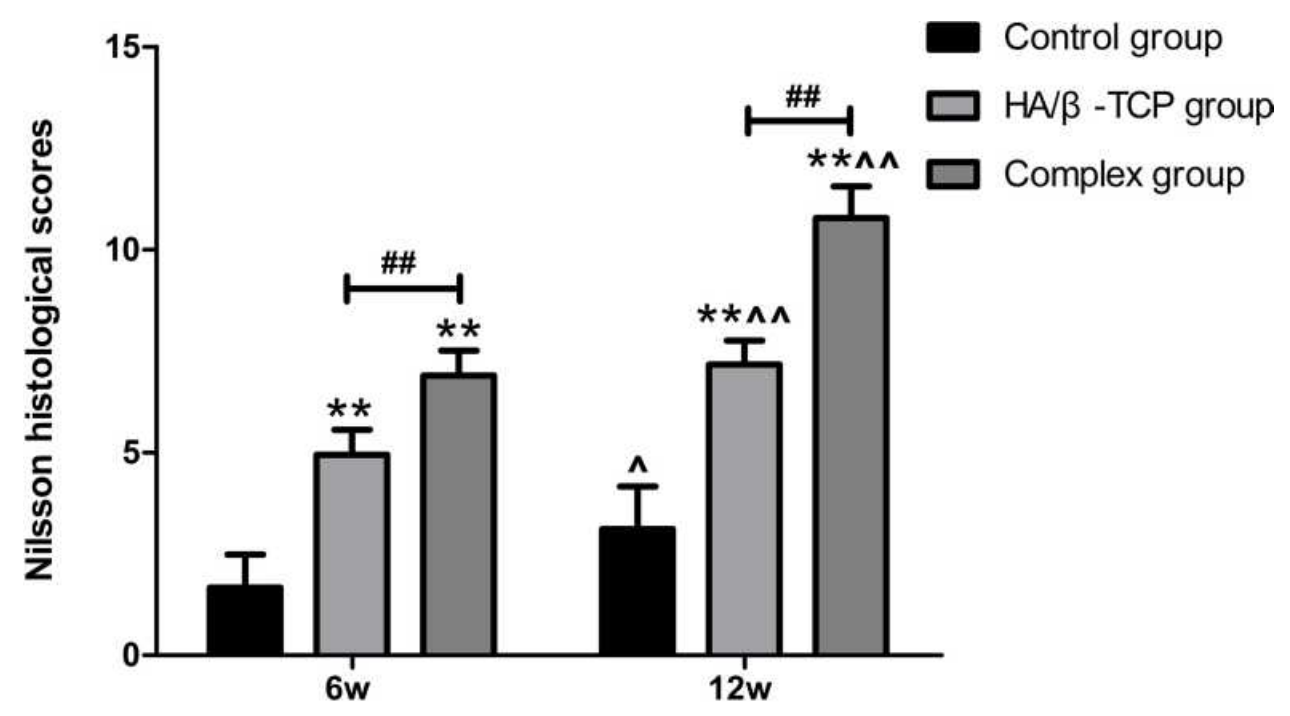

Figure 9 Nilsson histological scores for the samples from the three groups at 6 and 12 weeks after surgery $(n=6)$.

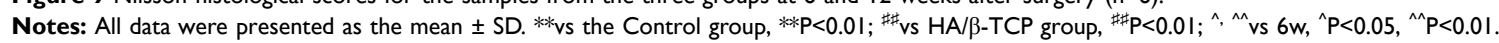

Abbreviation: $\mathrm{HA} / \beta-\mathrm{TCP}$, hydroxyapatite/ $\beta$ - tricalcium phosphate.

exhibited a significant downward trend, which gradually decreased to the normal level at 12 weeks after operation. However, it slowly decreased in the HA/ $\beta$-TCP group and control group and was still higher than the normal level at 12 weeks after operation. Like rifampin, rifapentine works by selective binding to Mycobacterium tuberculosis's DNA-dependent RNA polymerase, but not to the polymerase in mammalian cells. ${ }^{32}$ Therefore, it has excellent sterilizing activity. When the content of Mycobacterium tuberculosis in the lesions decreased, the inflammation was alleviated, and the secretion of CRP was reduced. ${ }^{33}$ It indirectly showed that the complex inhibited the bacterial infection.

To validate the tissue repair effects, test aimed to use the complex to repair bone defects of spinal TB was carried out. The macroscopical and histological evidence and statistical result of histological scores demonstrated that bone defects repair in the complex group showed better repair than the other two groups. The regeneration of bone tissue depends on blood supply. The migration and formation of stem cells or osteoprogenitor cells are important for bone tissue repair. ${ }^{34}$ These cells are transferred to the lesion site under the influence of various factors. Inflammatory factors, growth factors and chemokines at the lesion can promote osteogenic cell differentiation and the repair process. ${ }^{35}$ In the HA/ $\beta$-TCP group and Control group, due to the lack of anti-TB drugs, local inflammatory reactions persisted, and disordered release of various factors affected cell migration and osteogenic differentiation. ${ }^{36}$ In addition, a recent study shows that inflammatory cells could inhibit proliferation and synthesis of mineralized extracellular matrix of BMSCs. ${ }^{37}$ Therefore, local bone tissue in the HA/ $\beta$-TCP group and Control group could not be completely regenerated, but replaced by fibrous tissue.

In summary, the complex constructed of RPSMs and HA/ $\beta$-TCP demonstrated a long drug release time, with no significant inhibition of cell differentiation in vitro experiments. The effect of the complex on drug therapy and local tissue repair of spinal TB were verified by in vivo experiments. As the spinal TB model cannot control issues such as the severity of lesion, immunity to the disease and how the complex helped in clearing out the TB load, further research is needed to assess the long-term outcomes of such a treatment method.

\section{Conclusion}

The results of this study indicate that the complex not only has the ability of sustained-release drug and good cytocompatibility in vitro but also has the ability of anti-tuberculosis and good tissue repair effects in vivo, and thus provides a new method for clinical treatment of spinal TB.

\section{Acknowledgments}

This study is supported by the National Natural Science Foundation of China (Grant No. 81860394).

\section{Disclosure}

The authors report no conflict of interests in this work. 


\section{References}

1. World Health Organization. Global tuberculosis report 2020; 2020. Available from: https://www.who.int/tb/publications/global_report/ en/. Accessed January 29, 2021.

2. Xu Z, Wang X, Shen X, et al. One-stage lumbopelvic fixation in the treatment of lumbosacral junction tuberculosis. Eur Spine J. 2015;24 (8):1800-1805. doi:10.1007/s00586-015-3863-8

3. Ge Z, Wang Z, Wei M. Measurement of the concentration of three antituberculosis drugs in the focus of spinal tuberculosis. Eur Spine J. 2008;17(11):1482-1487. doi:10.1007/s00586-008-0778-7

4. Elmorsy E, Attalla SM, Fikry E, et al. Adverse effects of anti-tuberculosis drugs on HepG2 cell bioenergetics. Hum Exp Toxicol. 2017;36(6):616-625. doi:10.1177/0960327116660751

5. Yang TW, Park HO, Jang HN, et al. Side effects associated with the treatment of multidrug-resistant tuberculosis at a tuberculosis referral hospital in South Korea: a retrospective study. Medicine (Baltimore). 2017;96(28):e7482. doi:10.1097/MD.0000000000007482

6. Shive MS, Anderson JM. Biodegradation and biocompatibility of PLA and PLGA microspheres. Adv Drug Deliv Rev. 1997;28 (1):5-24. doi:10.1016/s0169-409x(97)00048-3

7. Rastogi N, Goh KS, Berchel M, Bryskier A. Activity of rifapentine and its metabolite 25-O-desacetylrifapentine compared with rifampicin and rifabutin against Mycobacterium tuberculosis, Mycobacterium africanum, Mycobacterium bovis and M. bovis BCG. J Antimicrob Chemother. 2000;46:565-570. doi:10.1093/jac/46.4.565

8. Alfarisi O, Alghamdi WA, Al-Shaer MH, Dooley KE, Peloquin CA. Rifampin vs. rifapentine: what is the preferred rifamycin for tuberculosis? Expert Rev Clin Pharmacol. 2017;10(10):1027-1036. doi:10.1080/17512433.2017.1366311

9. Biver E, Calmy A, Rizzoli R. Bone health in HIV and hepatitis B or C infections. Ther Adv Musculoskelet Dis. 2017;9(1):22-34 doi:10.1177/1759720X16671927

10. Kapasa ER, Giannoudis PV, Jia X, Hatton PV, Yang XB. The effect of RANKL/OPG balance on reducing implant complications. J Funct Biomater. 2017;8(4):42. doi:10.3390/jfb8040042

11. Liu Y, Duan D, Xin Y, et al. A review of the literature: antibiotic usage and its relevance to the infection in periodontal flaps. Acta Odontol Scand. 2017;75(4):288-293. doi:10.1080/ 00016357.2017.1295165

12. Liu Y, Zhu J, Jiang D. Release characteristics of bonelike hydroxyapatite/poly amino acid loaded with rifapentine microspheres in vivo. Mol Med Rep. 2017;16(2):1425-1430. doi:10.3892/mmr.2017.6747

13. Marques CF, Olhero SM, Torres PMC, et al. Novel sintering-free scaffolds obtained by additive manufacturing for concurrent bone regeneration and drug delivery: proof of concept. Mater Sci Eng C Mater Biol Appl. 2019;94:426-436. doi:10.1016/j. msec.2018.09.050

14. Wang Z, Song X, Yang H, Maimaitiaili A, Wang T. Development and in vitro characterization of rifapentine microsphere-loaded bone implants: a sustained drug delivery system. Ann Palliat Med. 2020;9(2):375-387. doi:10.21037/apm.2020.03.13

15. Nilsson OS, Urist MR, Dawson EG, Schmalzried TP, Finerman GA. Bone repair induced by bone morphogenetic protein in ulnar defects in dogs. J Bone Joint Surg Br. 1986;68-B(4):635-642. doi:10.1302/ 0301-620X.68B4.3733844

16. Wu P, Wang $\mathrm{XY}, \mathrm{Li} \mathrm{XG}$, et al. One-stage posterior procedure in treating active thoracic spinal tuberculosis: a retrospective study. Eur J Trauma Emerg Surg. 2015;41(2):189-197. doi:10.1007/ s00068-014-0421-8.

17. Rajasekaran S, Khandelwal G. Drug therapy in spinal tuberculosis. Eur Spine J. 2013;22(S4):587-593. doi:10.1007/s00586-012-2337-5

18. Guo X, Zhu X, Liu D, Gong Y, Sun J, Dong C. Continuous delivery of propranolol from liposomes-in-microspheres significantly inhibits infantile hemangioma growth. Int J Nanomed. 2017;12:6923-6936. doi:10.2147/IJN.S137634
19. Allison SD. Analysis of initial burst in PLGA microparticles. Expert Opin Drug Deliv. 2008;5(6):615-628. doi:10.1517/17425247.5.6.615

20. Huang J, Chen Z, Li Y, Li L, Zhang G. Rifapentine-linezolid-loaded PLGA microspheres for interventional therapy of cavitary pulmonary tuberculosis: preparation and in vitro characterization. Drug Des Devel Ther. 2017;11:585-592. doi:10.2147/DDDT.S127897

21. Zhang Z, Wu L, Li H, Long Z, Song X. Drug release characteristics and tissue distribution of rifapentine polylactic acid sustained-release microspheres in rabbits after paravertebral implantation. Iran Red Crescent Med J. 2016;18(11):e38661. doi:10.5812/ircmj.38661

22. Rathbone CR, Cross JD, Brown KV, Murray CK, Wenke JC. Effect of various concentrations of antibiotics on osteogenic cell viability and activity. J Orthop Res. 2011;29(7):1070-1074. doi:10.1002/ jor.21343

23. Zhang Z, Wang X, Luo F, et al. Effects of rifampicin on osteogenic differentiation and proliferation of human mesenchymal stem cells in the bone marrow. Genet Mol Res. 2014;13(3):6398-6410. doi:10.4238/2014.August. 25.3

24. $\mathrm{Li} \mathrm{H}$, Yue B. Effects of various antimicrobial agents on multi-directional differentiation potential of bone marrow-derived mesenchymal stem cells. World J Stem Cells. 2019;11(6):322-336. doi:10.4252/wjsc.v11.i6.322

25. Gamie Z, Tran GT, Vyzas G, et al. Stem cells combined with bone graft substitutes in skeletal tissue engineering. Expert Opin Biol Ther. 2012;12(6):713-729. doi:10.1517/14712598.2012.679652

26. Bassi G, Guilloton F, Menard C, et al. Effects of a ceramic biomaterial on immune modulatory properties and differentiation potential of human mesenchymal stromal cells of different origin. Tissue Eng Part A. 2015;21(3-4):767-781. doi:10.1089/ten.TEA.2014.0269

27. Mebarki M, Coquelin L, Layrolle P, et al. Enhanced human bone marrow mesenchymal stromal cell adhesion on scaffolds promotes cell survival and bone formation. Acta Biomater. 2017;59:94-107. doi:10.1016/j.actbio.2017.06.018

28. Thangavelu M, Narasimha RR, Adithan A, Chandrasekaran A, JongHoon K, Parvathaleswara ST. Reengineered graft copolymers as a potential alternative for the bone tissue engineering application by inducing osteogenic markers expression and biocompatibility. Colloids Surf B Biointerfaces. 2016;143:15-26. doi:10.1016/j. colsurfb.2016.03.021

29. Singh AK, Gupta UD. Animal models of tuberculosis: lesson learnt. Indian $J$ Med Res. 2018;147(5):456-463. doi:10.4103/ijmr. IJMR_554_18

30. Liu $\mathrm{P}$, Jiang $\mathrm{H}$, Li S, Lin $\mathrm{Z}$, Jiang J. Determination of anti-tuberculosis drug concentration and distribution from sustained release microspheres in the vertebrae of a spinal tuberculosis rabbit model. J Orthop Res. 2017;35(1):200-208. doi:10.1002/jor.23236

31. Yan L, Jiang DM, Cao ZD, et al. Treatment of Staphylococcus aureus-induced chronic osteomyelitis with bone-like hydroxyapatite/ poly amino acid loaded with rifapentine microspheres. Drug Des Devel Ther. 2015;9:3665-3676. doi:10.2147/DDDT.S84486

32. Dickinson JM, Mitchison DA. In vitro properties of rifapentine (MDL473) relevant to its use in intermittent chemotherapy of tuberculosis. Tubercle. 1987;68(2):113-118. doi:10.1016/00413879(87)90026-2

33. Sigal GB, Segal MR, Mathew A, et al. Biomarkers of tuberculosis severity and treatment effect: a directed screen of 70 host markers in a randomized clinical trial. EBioMedicine. 2017;25:112-121. doi:10.1016/j.ebiom.2017.10.018

34. Tam WL, Luyten FP, Roberts SJ. From skeletal development to the creation of pluripotent stem cell-derived bone-forming progenitors. Philos Trans R Soc Lond B Biol Sci. 2018;373(1750):20170218. doi:10.1098/rstb.2017.0218

35. Li C, Li G, Liu M, Zhou T, Zhou H. Paracrine effect of inflammatory cytokine-activated bone marrow mesenchymal stem cells and its role in osteoblast function. $J$ Biosci Bioeng. 2016;121(2):213-219. doi:10.1016/j.jbiosc.2015.05.017 
36. Wang $\mathrm{N}$, Zhou $\mathrm{Z}$, Wu T, et al. TNF- $\alpha$-induced NF- $\mathrm{kB}$ activation upregulates microRNA-150-3p and inhibits osteogenesis of mesenchymal stem cells by targeting $\beta$-catenin. Open Biol. 2016;6(3):150258. doi:10.1098/rsob.150258
37. Bastian OW, Croes M, Alblas J, Koenderman L, Leenen LPH, Blokhuis TJ. Neutrophils inhibit synthesis of mineralized extracellular matrix by human bone marrow-derived stromal cells in vitro. Front Immunol. 2018;9:945. doi:10.3389/fimmu.2018.00945

\section{Publish your work in this journal}

Infection and Drug Resistance is an international, peer-reviewed openaccess journal that focuses on the optimal treatment of infection (bacterial, fungal and viral) and the development and institution of preventive strategies to minimize the development and spread of resistance. The journal is specifically concerned with the epidemiology of

Submit your manuscript here: https://www.dovepress.com/infection-and-drug-resistance-journal antibiotic resistance and the mechanisms of resistance development and diffusion in both hospitals and the community. The manuscript management system is completely online and includes a very quick and fair peerreview system, which is all easy to use. Visit http://www.dovepress.com/ testimonials.php to read real quotes from published authors. 